ARTICLE

Received 6 Aug 2015 | Accepted 3 Nov 2015 | Published 10 Dec 2015

\title{
Mechanisms of assembly and genome packaging in an RNA virus revealed by high-resolution cryo-EM
}

\author{
Emma L. Hesketh ${ }^{1}$, Yulia Meshcheriakova ${ }^{2}$, Kyle C. Dent ${ }^{1, \dagger}$, Pooja Saxena ${ }^{2, \dagger}$, Rebecca F. Thompson ${ }^{1}$, \\ Joseph J. Cockburn', George P. Lomonossoff ${ }^{2} \&$ Neil A. Ranson ${ }^{1}$
}

Cowpea mosaic virus is a plant-infecting member of the Picornavirales and is of major interest in the development of biotechnology applications. Despite the availability of $>100$ crystal structures of Picornavirales capsids, relatively little is known about the mechanisms of capsid assembly and genome encapsidation. Here we have determined cryo-electron microscopy reconstructions for the wild-type virus and an empty virus-like particle, to $3.4 \AA$ and $3.0 \AA$ resolution, respectively, and built de novo atomic models of their capsids. These new structures reveal the C-terminal region of the small coat protein subunit, which is essential for virus assembly and which was missing from previously determined crystal structures, as well as residues that bind to the viral genome. These observations allow us to develop a new model for genome encapsidation and capsid assembly.

\footnotetext{
${ }^{1}$ Astbury Centre for Structural Molecular Biology, University of Leeds, Mount Preston Street, Leeds LS2 9JT, UK. ${ }^{2}$ Department of Biological Chemistry, John Innes Centre, Norwich Research Park, Colney, Norwich NR4 7UH, UK. † Present addresses: Life Science Division, Diamond Light Source, Harwell Science and Innovation Campus, Fermi Avenue, Didcot, Oxfordshire OX11 0QX, UK (K.C.D.); Department of Chemistry, Indiana University, 800 East Kirkwood Avenue, Bloomington, Indiana 47405-7102, USA (P.S.). Correspondence and requests for materials should be addressed to N.A.R. (email: n.a.ranson@leeds.ac.uk).
} 
A crucial step in virus assembly is the specific encapsidation of the genome. This is a particular challenge for single-stranded RNA viruses, as they must preferentially select their genomes from a high background of cellular mRNA. Owing to their importance as pathogens and their relatively simple, non-enveloped capsids, members of the order Picornavirales have been extensively studied. These viruses include members infecting vertebrates (poliovirus, hepatitis A virus), insects (deformed wing virus) and plants (cowpea mosaic virus (CPMV)) and $>100 \mathrm{X}$-ray structures of their capsids are available ${ }^{1}$. However, while these reveal the structure of the coat proteins in exquisite detail, they give very few clues about how RNA is packaged or how specificity is achieved. Despite many years of study on the replication cycle of the Picornavirales, remarkably little is known about the process of RNA encapsidation. For example, it is not known which type of coat protein aggregate is required for efficient RNA incorporation or what controls the specificity of RNA packaging ${ }^{2}$.

CPMV, the type member of the Comoviridae subfamily of the plant-infecting Secoviridae, has a bipartite, positive-sense, singlestranded RNA genome. The two segments, RNA-1 $(6 \mathrm{~kb})$ and RNA-2 (3.5 kb) (Fig. 1a), are separately encapsidated. Its icosahedral particles have a maximum diameter of $\sim 30 \mathrm{~nm}$ and are comprised of 60 copies each of a Large (L) and Small (S) coat protein (Fig. 1). L and $\mathrm{S}$ are processed from a single precursor polyprotein, the RNA-2-encoded VP60, by the action of the RNA-1-encoded $24 \mathrm{~K}$ proteinase. Particles containing the two different genomic RNAs can readily be purified (Fig. 1c). Crystallographic structures are available for three comoviruses ${ }^{3}$ : $\mathrm{CPMV}^{4,5}$, bean pod mottle virus (BPMV) ${ }^{6}$ and red clover mottle virus $^{7}$. Together, the $\mathrm{L}$ and $\mathrm{S}$ subunits comprise three $\beta$-barrel domains (two from L, one from S; Fig. 1d) corresponding to the three quasi-equivalent conformers of a $T=3$ icosahedral lattice. Comovirus capsids thus adopt a $\mathrm{p} T=3$ quasi symmetry (Fig. 1e), forming a particle with pronounced turrets formed from the $S$ subunit at the particle fivefold axes. Indeed, a penton of $\mathrm{L}$ and $\mathrm{S}$ subunits appears to be the basic building block for all Picornavirales capsids ${ }^{2}$. While the capsid structure is wellunderstood, the organization of encapsidated genomic RNA is not. In only one instance, the RNA-2-containing component of BPMV, can details of RNA structure be observed ${ }^{6}$, where density for 11 ordered ribonucleotides near the particle threefold axes was visualized ${ }^{6}$. This RNA forms a trefoil, and binds in a complementary depression on the inside of the capsid. Owing to icosahedral averaging it was impossible to deduce the RNA sequence, but the base composition was not random, and it was suggested that these sequences might be critical determinants for assembly or stability of capsids ${ }^{8}$.

The mechanisms by which RNA is selected and packaged are also poorly understood. The only portion of the CPMV capsid proteins currently implicated in RNA packaging is a segment of 24 amino acids at the $\mathrm{C}$ terminus of the $\mathrm{S}$ subunit ${ }^{9}$. This sequence is proteolytically cleaved during maturation without affecting particle stability or infectivity ${ }^{10}$, and is therefore missing from $\mathrm{X}$-ray structures of $\mathrm{CPMV}^{4}$. The suggestion that this region may promote interaction with RNA was supported by the fact that the S subunit loses its suppressor of host RNA silencing activity when the $\mathrm{C}$ terminus is removed ${ }^{11}$. However, from studies using a system that can produce RNA-free CPMV capsids in the absence of infection ${ }^{12}$ it is clear that this extension is also essential for capsid assembly itself ${ }^{13}$. Deletion of the entire 24 -amino acid sequence almost completely abolished empty particle formation. This effect could be partially reversed by substituting the 24 amino acids with 6 histidines, suggesting that basic amino acids in the $\mathrm{C}$-terminal extension may play a role in capsid assembly ${ }^{13}$.

In recent years, the Comoviridae, especially CPMV, have become a major focus of research in biotechnology. CPMV infects legumes and Nicotiana benthamiana, and grows to extremely high titres ( $>1 \mathrm{~g} \mathrm{~kg}^{-1}$ of leaf tissue). Like many plant viruses, it is also exceptionally stable in the environment. CPMV capsids can now be both genetically and chemically manipulated, and insertion of foreign sequences into surface loops is routine ${ }^{13-15}$. CPMV is therefore being developed for many biotechnology applications, including biosensors, targeted nano-containers for drug delivery, nanomaterials, imaging agents and as a platform for novel vaccine development ${ }^{16-27}$. However, wild-type CPMV has evolved to package its genome, reducing the potential for loading with heterologous cargoes. To overcome this problem, an empty virus-like particle (eVLP) technology has been developed. Expression of the precursor of L and S subunits (VP60) in plants using an Agrobacterium-based, pEAQ vector system ${ }^{28}$ (Fig. 1a) a

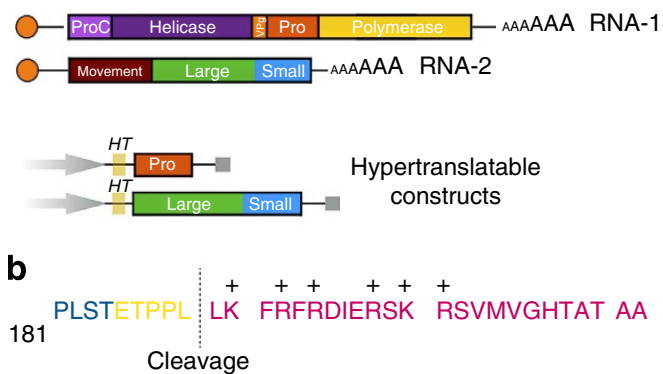

c

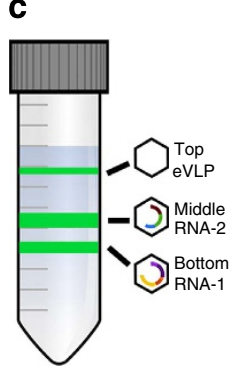

d

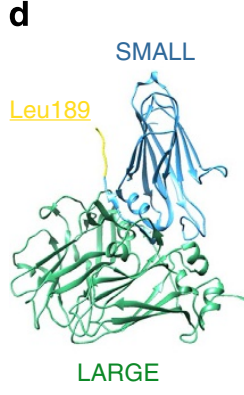

e

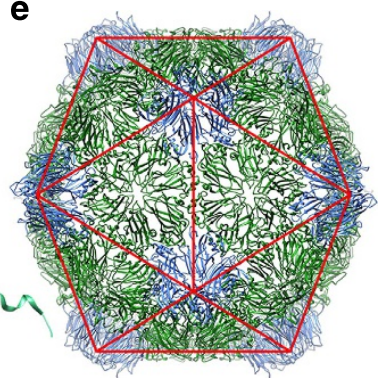

Figure 1 | An introduction to CPMV. (a) Schematic of the Cowpea mosaic virus (CPMV) bipartite, positive-sense single-stranded RNA genome. RNA-1 is $6 \mathrm{~kb}$ in length and contains the non-structural genes, while RNA-2 is $3.5 \mathrm{~kb}$ in length and contains the sequence encoding the large coat protein ( $L$ subunit) and small coat protein ( $S$ subunit). Hypertranslatable constructs (HT) separately expressing the viral proteinase and the precursor of the $L$ and $S$ subunits are used for production of eVLPs using the pEAQ vector system. (b) The sequence of S subunit amino acids $180-213$. The C-terminal 24 amino acid segment of S subunit is cleaved following assembly, and is coloured magenta. The cleavage site between Leu189 and Leu190 is shown. This region of the polypeptide is highly positively charged and the positive amino acids are indicated. (c) Schematic of CPMV density purification. RNA-1 containing CPMV sediments at the bottom of a Nycodenz gradient (CPMV-B), RNA-2-containing CPMV (CPMV-M) sediment in the middle and empty CPMV particles sediment at the top of the gradient (CPMV-T). CPMV-T particles are the natural equivalent to empty virus-like particles (eVLPs). (d) X-ray crystal structure of the asymmetric unit of CPMV (PDB $1 N Y 7^{4}$ ), coloured as above. The C-terminal amino acid of the S subunit (leucine 189) is indicated. (e) Icosahedral organization of CPMV (using PDB $1 N Y 7^{4}$ ). Each icosahedral particle is comprised of 60 copies of both the $L$ subunit and the $S$ subunit. $A$ view down the twofold axis is shown. 
produces large quantities of essentially insoluble protein ${ }^{12}$. Particle formation only occurs on co-expression with the viral $24 \mathrm{~K}$ proteinase, allowing normal proteolytic processing of VP60 (ref. 12). The result is efficient release of mature coat protein subunits and large quantities of eVLPs, which can be loaded with metal and metal oxide provided the C-terminal residues of the $\mathrm{S}$ subunit are first proteolytically removed ${ }^{13}$. Recently, modification of the eVLP surface and particle loading with metal ions $\left(\mathrm{Co}^{2+}, \mathrm{Fe}^{2+}\right.$ and $\left.\mathrm{Fe}^{3+}\right)$ have been achieved simultaneously for the first time ${ }^{29}$, and this may herald attempts to develop targeted therapeutics. Furthermore insertion of integrin-binding sequences into the surface loops of an eVLP has allowed targeting of eVLPs to human endothelial cells (Meshcheriakova et al., unpublished).

However, despite much research on CPMV, its true potential in biotechnology may not be realized until we achieve a full understanding of the mechanisms that underlie capsid assembly and encapsidation of genomic RNA (or other cargo). It is not yet, for example, possible to package heterologous RNA or DNA. To gain insights into CPMV biogenesis, we have determined high-resolution cryo-electron microscopy (cryo-EM) structures for the CPMV eVLP, in which the C-terminal extension to the $\mathrm{S}$ subunit is visible, to 3.0 - $\AA$ resolution. We have also determined the structure for wild-type CPMV containing its larger genomic RNA at $3.4 \AA$ resolution, where density for genomic RNA is resolved. The resolution of both maps is sufficient to allow de novo atomic models to be built. Furthermore, the availability of a system for producing capsids in the absence of infection has enabled us to use mutagenesis to explore the role of those amino acids predicted by the models to be involved in particle formation, and to discriminate between these and residues involved in RNA packaging. This combined analysis has enabled us to develop a new model for RNA recognition and capsid assembly.

\section{Results}

Cryo-EM structure determination. Although the C-terminal extension to the S subunit is implicated in capsid assembly and RNA packaging, understanding these roles has been difficult because the normal maturation of the RNA-filled capsid involves its cleavage and dissociation. As a result no structural information for these residues is currently available. To address this deficiency, we decided to examine the structure of the CPMV eVLP particle. Crucially, not only are such eVLPs completely lacking in any encapsidated RNA, they also undergo C-terminal cleavage more slowly than wild-type virions, raising the possibility that we could determine the structure of an eVLP that retains the C-terminal segment using cryo-EM and single particle image processing. We therefore collected a cryo-EM data set for CPMV eVLP comprising $\sim 1,150$ micrographs collected on an FEI Titan Krios microscope, using a direct electron detector (for details of data collection and image processing see Methods). Each micrograph was recorded as an exposure movie consisting of 35 frames, which were computationally corrected for microscope drift and beam-induced movement ${ }^{30}$. Particles were selected semi-automatically ${ }^{31}$, and a data set of $62.5 \mathrm{k}$ particles was assembled. Iterative rounds of two-dimensional (2D) and 3D classification were then used to select a homogeneous subset of 4,998 particles for 3D structure refinement (see Methods). The resulting final density map was sharpened using an empirically derived $B$-factor of $-74.6 \AA^{2}$ to $3.04 \AA$ resolution (Fig. 2a; EMD-3014).

Wild-type, infectious CPMV particles containing RNA-1 (bottom fraction; CPMV-B) were collected from the bottom of a Nycodenz gradient, dialysed to remove the Nycodenz, and used for cryo-EM data collection. A data set of $\sim 1,750$ electron micrographs was collected on the same microscope and detector as described above. Particles were selected automatically, generating a total data set of $\sim 72 \mathrm{k}$ particles. A homogeneous subset $(4,331)$ of these particles was selected and used to determine a $3 \mathrm{D}$ reconstruction. The final structure for CPMV-B was sharpened using an empirically derived $B$-factor of $-107.6 \AA^{2}$ to a final resolution at $3.44 \AA$ (Fig. 2b; EMD-3013). It should be noted that the initial starting model for the eVLP structure was a sphere with a radius of $\sim 155 \AA$. The CPMV-B structure used the eVLP model filtered to $60 \AA$ A resolution. No information from the existing X-ray structure whatsoever was therefore used to generate either of the structures presented here.

Atomic model building. As shown in Fig. 2, the resolution of both eVLP and CPMV-B maps is high enough to clearly resolve amino acid side chains in the density. We therefore decided to build de novo atomic models into the EM density rather than rely on existing atomic models for the CPMV capsid proteins (PDB $\left.1 \mathrm{NY} 7^{4}\right)$. We started with the higher resolution eVLP map, and built the polypeptide chain of a single copy of both the L and S subunit using $\operatorname{Coot}^{32}$. This preliminary model was then iteratively refined and rebuilt using REFMAC5 $5^{33}$ and $\operatorname{Coot}^{32}$ to progressively improve model quality. The resulting model contained information for the majority of the polypeptide sequence, critically including a 13-residue segment in the C-terminal region of $S$ subunit that had never been previously visualized. The refined eVLP atomic model was then docked into the 3.4- $\AA$ CPMV-B map. Residues in the eVLP atomic model for which no density was observed for CPMV-B were deleted (residues 190-202 in S subunit) and amino acids resolved in CPMV-B but not eVLP were added and modelled (residues 184-189 in the S subunit). This preliminary (for the CPMV-B structure) model was then again iteratively refined in REFMAC5 to give the final model presented in Fig. $2 \mathrm{~b}$.

The structure of the $\mathrm{C}$-terminal extension to the $\mathrm{S}$ subunit. The existing structural information for the CPMV capsid ${ }^{3,4}$ show the C terminus of S subunit after cleavage (ending at residue 189) in an extended conformation running across the exterior surface of the capsid towards a cleft between the $S$ subunits that form the turret at an icosahedral fivefold vertex. This is precisely the conformation we see in our CPMV-B structure (see the yellow segment in Fig. 3a), but in the eVLP map we see additional density in this cleft that does not match the previously deposited structure. The density that would correspond to residues 184-189 in the $\mathrm{C}$ terminus is very weak suggesting this segment is poorly ordered in the particle in solution (see the yellow segment in Fig. $3 b$ ), and we have not been able to build a convincing model into this region of the map. However, it is clear that the polypeptide chain takes a steeper path along the edge of the cleft than it does once C-terminal cleavage (between residues 189 and 190) has occurred (comparison of yellow segments in Fig. 3a,b). The C-terminal segment then becomes ordered once more, and we see density corresponding to Leu190 to Arg202, residues absent from previous structures. A loop runs from the top of the $\mathrm{S}$ subunit back into the cleft between subunits, before forming two turns of $\alpha$-helix running out of the cleft towards the bulk solvent (see magenta segment Fig. 3b). The bottom of this segment appears to be very well-ordered, with clear density for side chains that make intimate contacts to the neighbouring $\mathrm{S}$ subunit around the penton (Fig. 3c). The density then becomes disordered once more, with Arg202 as the last ordered residue, suggesting that the $11 \mathrm{C}$-terminal residues are 

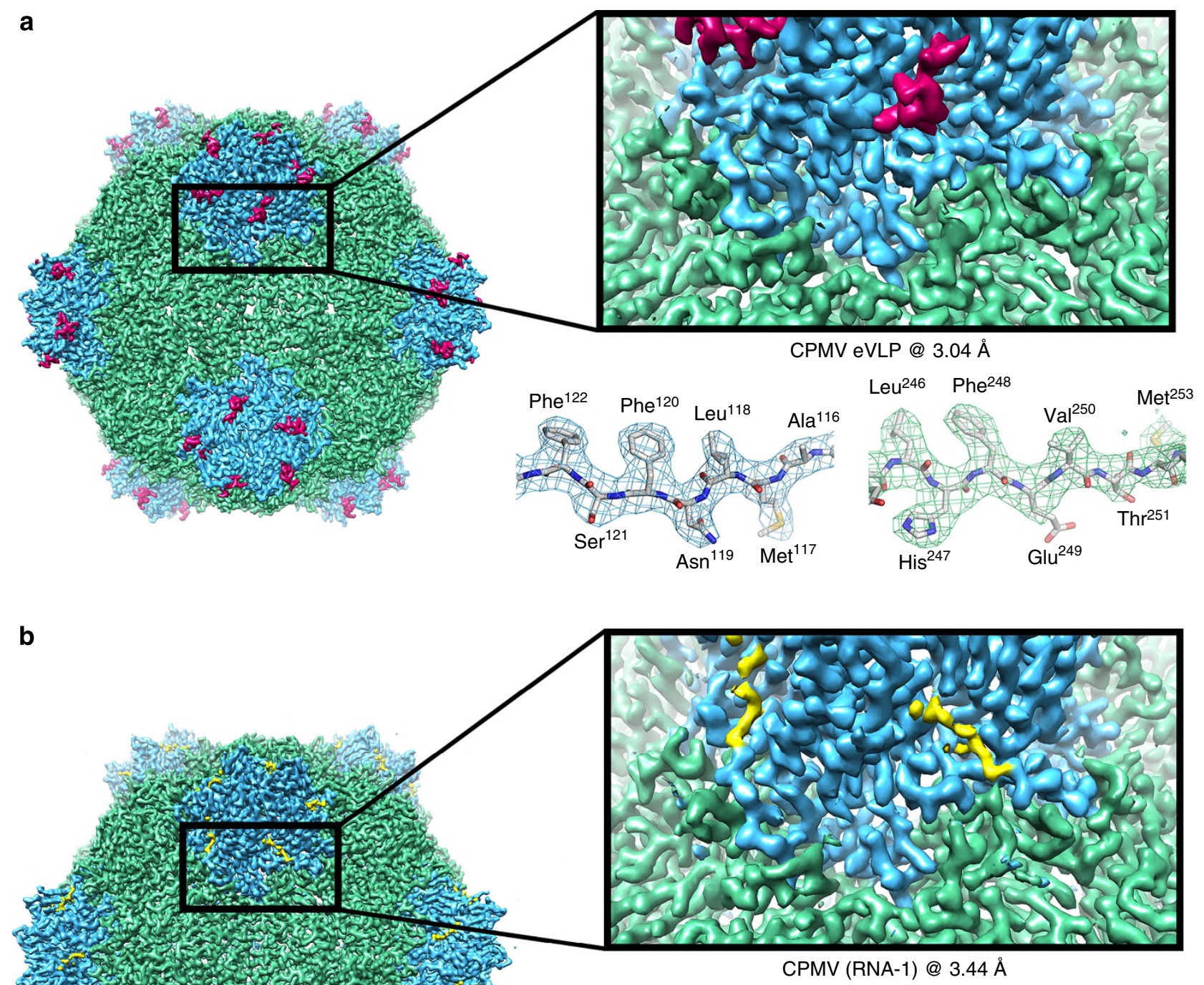

CPMV (RNA-1) @ $3.44 \AA$
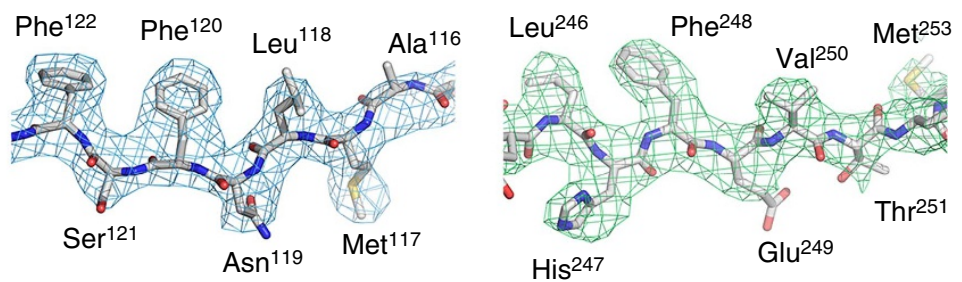

Figure 2 | Cryo-EM structures of eVLP and CPMV-B. (a) EM density map of CPMV empty virus-like particle (eVLP) determined by cryo-EM to $3.04 \AA$ resolution (EMDB-3014). The $L$ subunit is shown in green, the $S$ subunit in blue and the additionally visualized 13 amino acids in the $C$-terminal region of the $S$ subunit in magenta. On the right hand side, a zoomed-in view of the boundary between $L$ and $S$ subunits is shown. The density for an individual $\beta$ strand is shown in a mesh representation with the EM-derived atomic model within, showing clear resolution of large and small side chains. (b) Identical views as in $\mathbf{a}$, but showing the EM map of CPMV containing RNA-1 (CPMV-B) to $3.44 \AA$ resolution (EMDB-3013).

disordered in solution. Intriguingly, this tallies with functional observations that while truncation of the C-terminal segment by up to 11 residues are tolerated, larger truncations (12 residues or more) dramatically reduce the yield of intact eVLPs (see Supplementary Fig. 1 and Supplementary Table 1).

The role of the C-terminal extension. The ordered C-terminal segment described for the first time here forms an intimate network of interactions with the neighbouring $S$ subunit around the pentameric ring that forms the fivefold vertex of the particle. It is clear from the structure that hydrophobicity plays a central role in this network. Shown in Fig. 4a is the EM-derived atomic model for the eVLP represented as a surface, and coloured according to the hydrophobicity of the corresponding amino acid residues involved (see legend of Fig. 4 for details). Two phenylalanine residues in the C-terminal segment (F192 and F194) are well-resolved and appear to bind to a large hydrophobic patch on the body of the neighbouring $S$ subunit. To test the importance of these interactions, we made mutations in the $\mathrm{S}$ subunit sequence and analysed their effects on both eVLP assembly and RNA packaging by the virus. While F192W has little discernable effect on eVLP assembly, it dramatically reduces the efficiency of RNA packaging, resulting in 
a

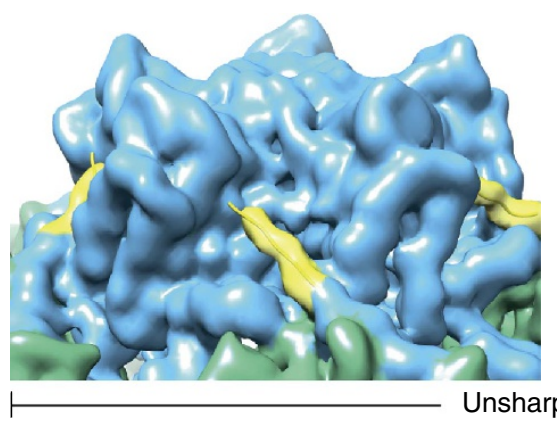

b

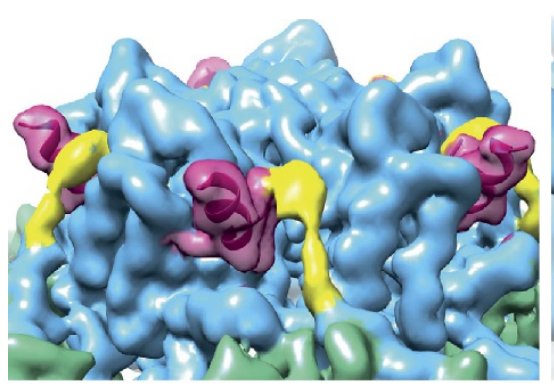

C

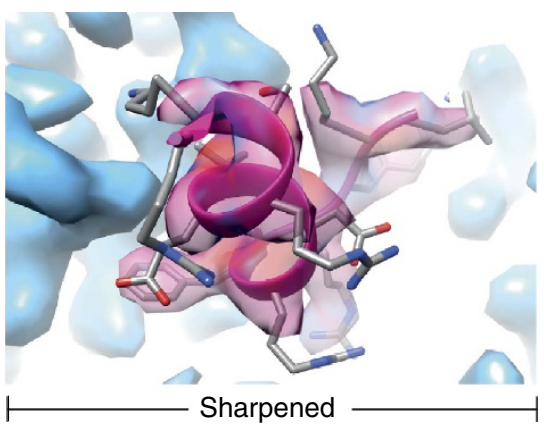

Figure 3 | The structure of the C-terminal extension of the $\mathbf{S}$ subunit. (a) EM density map of the unsharpened CPMV-B map with colours as described previously. In yellow is the $C$ terminus of CPMV-B (amino acids 184-189), which follows the same path as the current atomic model (PDB $1 N Y 7^{4}$ ). The final C-terminal amino acids (190-213) are missing from both the crystal structure and the CPMV-B EM density map. (b) EM density map of the unsharpened eVLP map with colours as described previously. The density corresponding to amino acid 184-189 is coloured yellow. Although this section of the EM density is too weak to allow a polypeptide backbone to be built, we can clearly see this portion of the C-terminal moves in the eVLP map compared with the CPMV-B map (see yellow segment in a). Coloured magenta is the newly resolved 13-amino acid residue (190-202 in the S subunit). (c) Zoomed-in version of the C-terminal extension from the sharpened EM density. The new atomic model is shown inside.
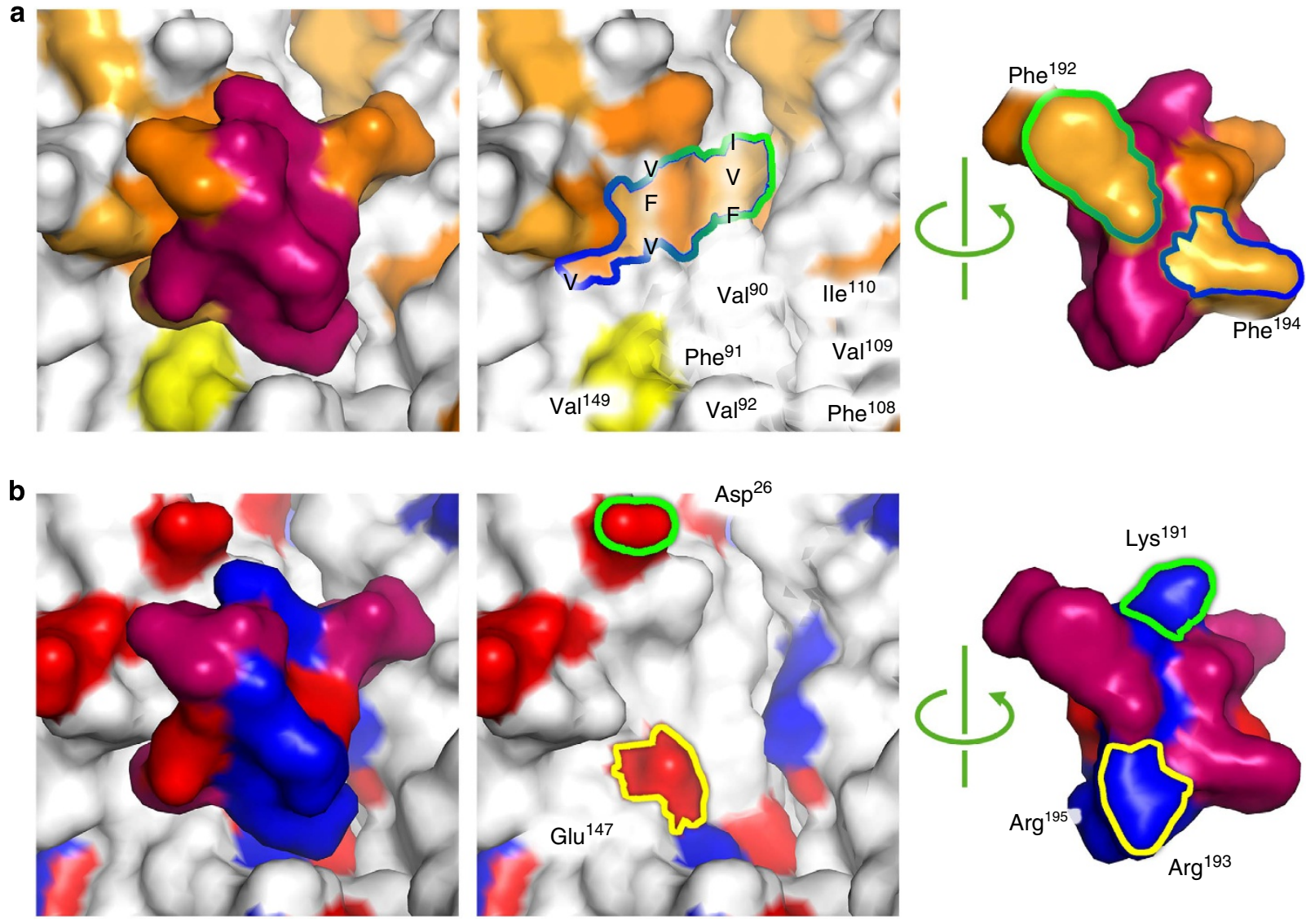

Figure 4 | Interactions between the C-terminal extension and the neighbouring $\mathbf{S}$ subunit. (a) EM-derived atomic model for the eVLP S subunit and C-terminal extension represented as a surface model and coloured according to hydrophobicity $(\mathrm{I}, \mathrm{L}$ and $\mathrm{V}$ : orange; $\mathrm{G}, \mathrm{A}$ and $\mathrm{F}$ : pale orange; $\mathrm{C}$ and $\mathrm{M}$ : yellow). The middle panel shows the surface of the $S$ subunit with the $C$-terminal extension removed, and the right panel shows the surface of the C-terminal extension that interacts with the $S$ subunit. Hydrophobic residues are labelled. (b) As in a coloured according to charge (red is negative, blue is positive). The charged residues from $\mathrm{S}$ subunit are labelled. All residues in Fig. 4 are from the $\mathrm{S}$ subunit.

large numbers of empty capsids and systemic movement of the virus in the plant does not occur (Supplementary Table 2 and 3; Fig. 5a). Mutation of the matching hydrophobic surface on the S subunit itself (for example, V109W) has even more profound effects, preventing assembly of particles (Fig. 5b). However, the network of interactions is complex, as mutation of the other phenylalanine residue (for example, F194W) has little discernable effect other than a slightly reduced particle yield.

Despite the extensive nature of the hydrophobic surface on both C-terminal extension and the S subunit surface to which it binds, a number of charged residues also appear to play a key 
a

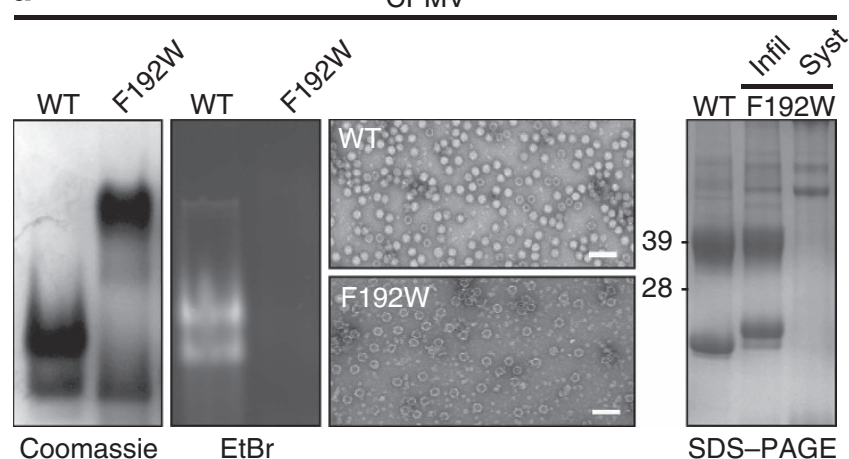

role. The C-terminal segment is highly basic, and side-chain density for two arginine residues is visible at the bottom of the cleft (R193 and R195; see Fig. 4b). R193 is particularly well-ordered and forms a salt-bridge to E147, again in the neighbouring $S$ subunit. To test the importance of this interaction, we mutated these residues and assayed for eVLP assembly and viral encapsidation of RNA in vivo. Both R193D and E147R are completely unable to assemble, while the double mutant R193D/E147R, which preserves the salt-bridge but swaps its directionality, is almost indistinguishable from the wild type (Fig. 5c). R195G is similar to wild-type in terms of assembly, suggesting that it is the R193-E147 salt-bridge that is crucial for assembly (Supplementary Table 2).

Interactions between the protein capsid and genomic RNA. The way in which eVLPs are expressed means that no genomic RNA is present in the cell, so none can possibly be packaged. However, the eVLP has previously been shown to be devoid of plant cell $\mathrm{mRNA}^{13}$, including the recombinant message for either the viral coat proteins or proteinase, which are the two mRNAs that should have the highest sequence similarity to the genome ${ }^{12}$. Indeed, in the $3.0 \AA$ structure of the eVLP there is no EM density that can be attributed to anything other than capsid proteins, which together with their very low A260/280 ratio and lack of ethidium bromide staining in agarose gels ${ }^{13}$ strongly suggest that the eVLP particles are devoid of RNA.

By contrast, the wild-type CPMV-B particle has packaged the full-length, 6-kb single-stranded RNA-1, and as expected we see significant extra density inside the capsid that we ascribe to this packaged RNA genome. However, the $B$-factor correction used to sharpen the map and reveal high-resolution features such as amino acid side chains acts as a strong high-pass Fourier filter, removing low-resolution features in the map such as poorly ordered molecular components like the genomic RNA. Shown in Fig. $6 \mathrm{a}$ is a 40 - $\AA$-thick central slab through the unsharpened CPMV-B map (at $3.63 \AA$ resolution; the unsharpened map is also included in the deposition for EMDB-3013). As seen in the cryo-EM structures of several single-stranded RNA viruses ${ }^{34,35}$, the RNA appears as concentric shells of density. It must be noted that this density is an icosahedrally averaged picture of an a

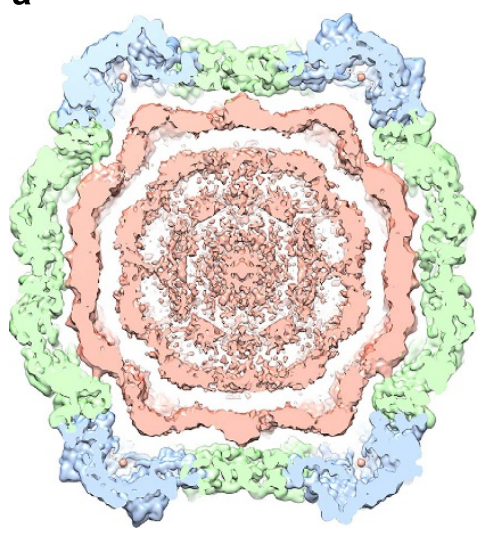

b

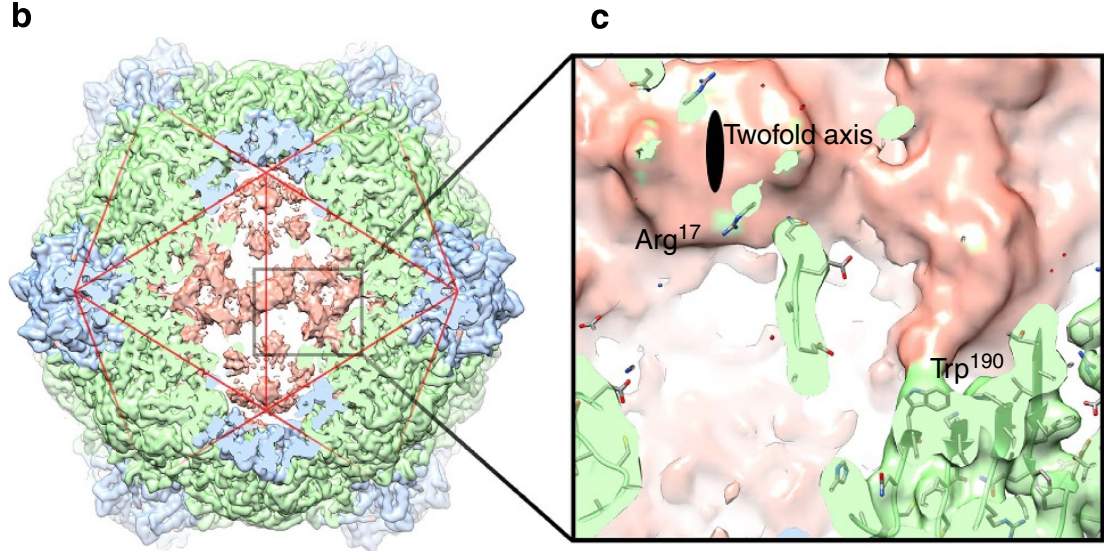

Figure 6 | Density for RNA-1 in the CPMV-B structure. (a) A 40- $\AA$ thick central slab through the unsharpened CPMV-B map (at $3.63 \AA$ resolution; unsharpened map is also included in the deposition for EMDB-3013, suggested contour level 0.015). The extra density ascribed to RNA is pink. Viral coat proteins coloured as described previously. (b) The strongest density for RNA is found beneath the capsid twofold axis, a binding site formed at the interface between two adjacent pentons. (c) Close up of viral RNA-protein interactions, demonstrating two major bridges of density between the viral RNA and the protein capsid. The density bridges are consistent with the involvement of W190 and R17 (both from the L subunit) in RNA binding. 
a

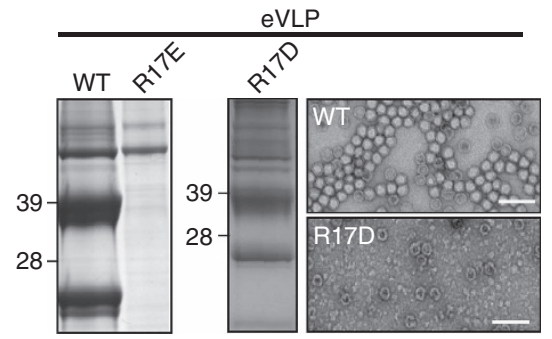

b

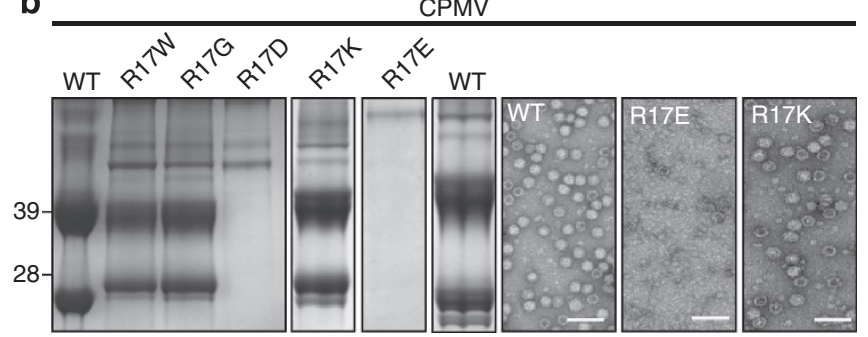

c

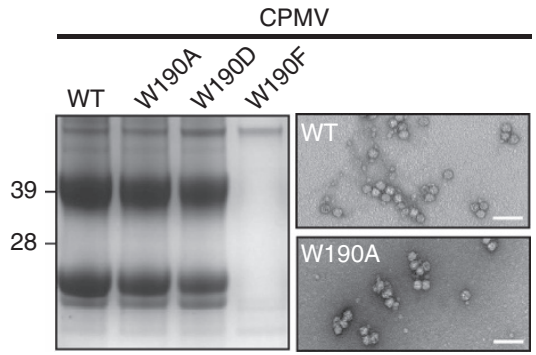

Figure 7 | Residues involved in encapsidation of genomic RNA.

(a) SDS-PAGE and negative stain EM showing the R17D mutation in eVLP has little effect on capsid assembly, however, R17E completely abolishes it. EM images of the WT eVLP show different stain penetration depending on the presence or absence of the $C$ terminus, with stain failing to penetrate complexes with an intact $C$ terminus. The preparation in the $E M$ is completely RNA free. (b) SDS-PAGE and negative stain EM showing R17 mutants in the L subunit. R17D and R17E abolish capsid assembly. Some flexibility is, however, tolerated as R17W, R17G and R17K all behave similarly to WT virus. (c) SDS-PAGE and negative stain EM showing W190 mutants in the $L$ subunit. W190F abolishes RNA binding and particle assembly. However, W190A and W190D are indistinguishable from WT. All scale bars, $100 \mathrm{~nm}$.

asymmetric RNA molecule, so precise structural interpretation is impossible. However, several observations can be made. First, the shells of density have a thickness of $\sim 20 \AA$, and are $\sim 20-25 \AA$ apart, consistent with the packing of duplex nucleic acids observed in other virus structures (for example, refs 34,36), suggesting that extensive base pairing occurs during encapsidation. The general form of the packaged RNA is dodecahedral rather than icosahedral (each is a different realization of 532 symmetry), with the strongest RNA feature in the map forming a truncated dodecahedral cage beneath the capsid shell. This is strongly reminiscent of RNA packaging in insect viruses of the Nodaviridae, where ordered genomic RNA is packaged as a dodecahedral cage in both X-ray and EM structures ${ }^{37,38}$. The strongest CPMV density is directly beneath the twofold symmetry axes of the capsid, which are formed by the interface between two adjacent pentons, implying that this is the site where RNA binding is strongest. The density fades out as the RNA extends away from the twofold axis towards the threefold junctions that form the vertices of the truncated dodecahedron. We see two major bridges of density between the capsid shell and the RNA density that are candidates for amino acid side chains,

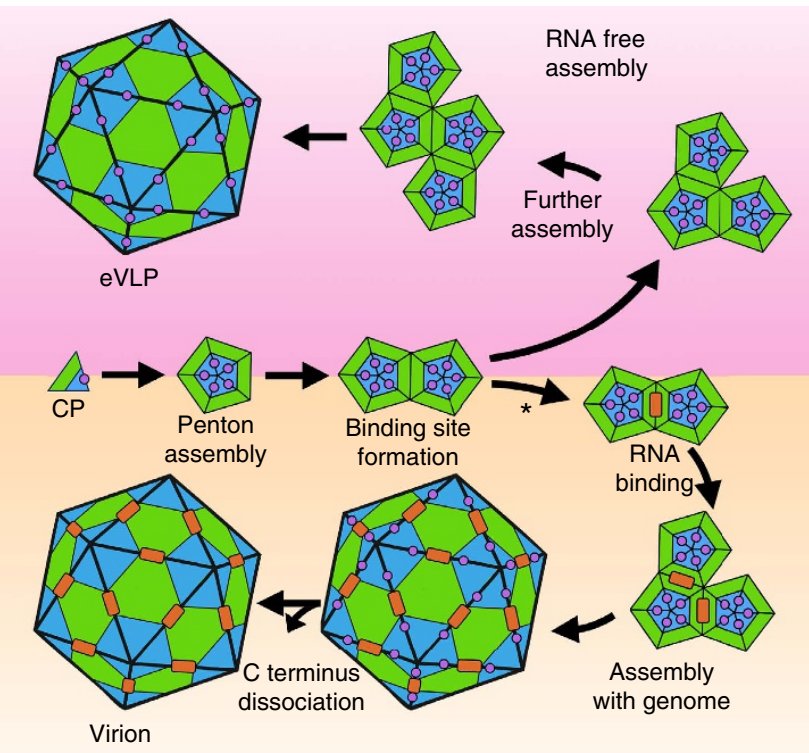

Figure 8 | A model for CPMV Assembly. The L (green) and S (blue) subunits associate to form a coat protein penton. The C-terminal extension (magenta circle) appears to act as a dab of 'molecular glue', stabilizing the formation of the penton due to extensive interactions with the neighbouring $\mathrm{S}$ subunit. In the absence of RNA (RNA free assembly shown in the top half of the diagram), C-terminal cleavage is slow, and eVLP particles are produced. After time, the C-terminal extension is lost from the $\mathrm{eVLP}$, suggesting that once an extensive protein:protein interaction network is formed in the capsid the eVLP structure is stable. In the presence of genomic RNA (assembly with genome shown in the bottom half of the diagram), assembly follows a different path. Initially, pentons are formed in the same way as in the eVLP, using the C-terminal extension for stability. Two pentons interact to form a twofold axis, and thus the RNAbinding site appears to exist at that position. Our model shows genomic RNA (represented as an orange rectangle) initially binds to the two pentons at this twofold axis, the first step in genome encapsidation. Following stepwise addition of pentons, the $\mathrm{C}$ terminus is rapidly cleaved implying that RNA occupancy is allosterically communicated through the structure to the outside of the capsid.

both from the L subunit, that directly interact with RNA (Fig. 6c). These are Arg17 and Trp190, with Trp190 being by far the strongest density feature connecting the capsid shell to the RNA.

To test the importance of these residues, we mutated each and examined the effect on eVLP assembly and RNA encapsidation in vivo (Fig. 7). Arg17 does indeed appear to be important for RNA packaging. Although R17D can be introduced into eVLPs with little effect, R17E substantially reduces eVLP capsid assembly (Supplementary Table 2; Fig. 7a). In wild-type virus, R17E and R17D abolish RNA packaging and substantially reduce capsid assembly (Supplementary Table 3; Fig. 7b). R17W, R17G and $\mathrm{R} 17 \mathrm{~K}$ are all indistinguishable from wild-type virus (Supplementary Table 3; Fig. 7b), with identical yield and systemic transport in plants, suggesting that the some degree of flexibility in the nature of the residue is tolerated. Similarly, mutations of W190A or W190D are both indistinguishable from wild-type virus, while W190F abolishes both RNA binding and capsid assembly (Supplementary Table 3; Fig. 7c).

\section{Discussion}

The cryo-EM structures presented here provide new insights into the structure of viruses in the Comoviridae. They demonstrate the ability of cryo-EM structures to guide site-directed mutagenesis 
that can begin to pick apart the molecular mechanisms that govern capsid assembly and genome packaging, providing the basis for a new model of CPMV particle assembly.

The C-terminal region of the $\mathrm{S}$ subunit, despite its importance for particle formation, is normally lost during virus maturation. The novel structure of this region presented here therefore reveals for the first time an extensive protein:protein interface that combines both hydrophobicity and electrostatic interactions that are important for capsid assembly and RNA encapsidation. Many questions remain, but the C-terminal extension occupies the cleft between the small coat protein subunits that form the pentameric capsomere from which the virus assembles (Fig. 8). While we do not resolve the residues that connect the C-terminal structure to the body of the S subunit (residues 184-189), we do see extensive interactions to the neighbouring subunit around the pentameric ring. The C-terminal extension may act therefore as a dab of 'molecular glue' stabilizing the structure of the penton of L and S subunits. In the absence of genomic RNA, this stabilization appears to be sufficient to allow formation of the intact eVLP. The eVLP does lose its C-terminal extension over time, implying that the stability gained from making the many protein:protein interactions formed during capsid assembly is sufficient to make the eVLP structure stable. In this context, the role of the C-terminal extension would in fact precisely fit the definition of a molecular chaperone or a scaffold protein, enhancing the efficiency of folding/assembly reactions without becoming a part of the final structure.

In the presence of genomic RNA, however, assembly takes a very different path. If the $C$ terminus stabilizes the CPMV penton, then the next step in building a capsid is the interaction between two pentons, which forms the first twofold axis of the growing particle. This is precisely the position at which our EM density suggests there is the strongest interaction with the genomic RNA. We suggest therefore that the C-terminal extension promotes the formation of the RNA-binding site itself, the first step in encapsidating the genomic RNA (Fig. 8; see asterisk). This is in contrast to previous models of BPMV assembly where RNA binding is proposed to occur at particle threefold axes ${ }^{39}$. However, the rapid cleavage of the $\mathrm{C}$ terminus in such conditions suggests that RNA binding must be allosterically communicated through the structure to the binding site of the $\mathrm{C}$ terminus on the outside of the capsid. Furthermore, the lack of obvious mixed electrophoretic forms that would reveal a mixture of cleaved/uncleaved subunits suggests that cleavage is strongly cooperative within a particle. The precise details of this communication remain unresolved, but the network of interactions is clearly complex. Mutation of residues on the inside of the capsid that directly interact with RNA prevent capsid assembly (for example, W190F in the large subunit). Conversely, residues on the outside of the capsid that were predicted to disturb protein:protein interactions allow capsid assembly but prevent RNA encapsidation (for example, F192W). Further structural studies on these mutants will likely help to elucidate the fine detail of these interactions.

Table 1 | Particle numbers at each stage in image processing.

\begin{tabular}{lcc} 
& eVLP & CPMV-B \\
\hline Autopicking & 62,514 & 72,061 \\
Particle sorting & 52,970 & 65,346 \\
2D classification & 39,800 & 48,189 \\
3D classification & 4,998 & 4,331
\end{tabular}

eVLP, empty virus-like particle; CPMV-B, cowpea mosaic virus $B ; 2 D$, two-dimensional; $3 D$, three-dimensional.
All members of the Comoviridae have a similar, cleavable C-terminal extension to their small coat protein subunit, although its length and sequence are highly variable. For example, in BPMV the sequence is strongly negatively charged in contrast to the positive charge found in CPMV. The structures presented here help to explain this diversity, because sequence changes in the C-terminal extension could easily be compensated for by surface mutations on the small subunit itself, as we show in the viability of the R193D/E147R mutant, which makes CPMV more BPMV-like in this regard. This idea is also consistent with observations that replacing the CPMV extension with the equivalent region from BPMV results in a virus with similar properties to one from which the $\mathrm{C}$-terminal residues have been deleted $^{11}$.

Although our studies provide an excellent view of capsid-RNA interactions in assembled particles, they do not, as yet, provide insights into how the specificity of RNA packing is achieved; this remains a goal of future structural studies in this system. The prevailing view in Picornavirales research is that RNA encapsidation is tightly linked to RNA replication. The paradigm is thus that specificity is a function of protein:protein rather than protein-RNA interactions, and that nucleotide sequence specificity is apparently unimportant. Our finding that the C-terminal 24 amino acids of the $\mathrm{S}$ subunit are involved in stabilizing the coat protein penton fits into this paradigm. The stabilization of the penton and the resulting promotion of interactions between pentons explains how this region can promote RNA encapsidation despite this sequence lying on the external surface of the assembled particle. Similar sequences are not found in other plant RNA viruses, perhaps because they commonly interact with their genomes via charged $\mathrm{N}$-terminal arms that are missing in the comoviruses. However, conceptually the role of the C-terminal sequence may be similar to the effect seen in Hepatitis A, an animal picornavirus, where the uncleaved $2 \mathrm{~A}$ peptide at the

Table 2 | Refinement and model statistics for atomic models built de novo into eVLP and CPMV-B EM density.

\begin{tabular}{|c|c|c|}
\hline & eVLP & CPMV-B \\
\hline \multicolumn{3}{|l|}{ Data collection } \\
\hline Particles & 4,998 & 4,331 \\
\hline Pixel size ( $\AA$ per pixel) & 1.04 & 1.04 \\
\hline Defocus range $(\mu \mathrm{m})$ & $0.5-5.0$ & $0.5-8.0$ \\
\hline Voltage $(\mathrm{kV})$ & 300 & 300 \\
\hline Dose $\left(e^{-} \operatorname{per} \AA^{2}\right)$ & 45 & 45 \\
\hline \multicolumn{3}{|l|}{ EM refinement } \\
\hline Final resolution $(\AA)$ & 3.04 & 3.44 \\
\hline Experimental $B$-factor $\left(\AA^{2}\right)$ & -74.6 & -107.6 \\
\hline EMDB accession number & EMD-3014 & EMD-3013 \\
\hline \multicolumn{3}{|l|}{ r.m.s. deviations } \\
\hline Bonds $(\AA)$ & 0.0077 & 0.0108 \\
\hline Angles $\left(^{\circ}\right)$ & 1.2957 & 1.5319 \\
\hline \multicolumn{3}{|l|}{ Model validation } \\
\hline R factor & 0.2207 & 0.2909 \\
\hline \multirow[t]{2}{*}{ Molprobity score } & 1.61 & 1.76 \\
\hline & (100th percentile) & (100th percentile) \\
\hline \multirow[t]{2}{*}{ Clashscore (all atoms) } & 0.81 & 1.52 \\
\hline & (100th percentile) & (100th percentile) \\
\hline Good rotamers (\%) & $96.3 \%$ & $96.5 \%$ \\
\hline PDB ID & $5 a 33$ & $5 a 32$ \\
\hline
\end{tabular}


C terminus of VP1 strongly promotes capsid assembly ${ }^{29}$. It also offers an explanation as to why this sequence can act to suppress RNA silencing when part of the $S$ subunit ${ }^{11}$ but not when it is fused to other proteins such as green fluorescent protein (Cañizares and Lomonossoff, unpublished), as the C-terminal extension's role is in chaperoning the formation of the silencingactive surface rather than silencing itself. However, given that the presence of the RNA accelerates the rate of C-terminal cleavage, it is plausible that both the $\mathrm{C}$ terminus and the genomic RNA may co-chaperone the assembly of the nascent virus particle.

\section{Methods}

\section{CPMV-B and CPMV eVLP purification. Infection of $N$. benthamiana with} CPMV was initiated by agroinfiltration of 3-week-old plants with pBinP-S1NT and pBinP-S2NT ${ }^{40}$. Wild-type eVLPs were produced either by co-infiltration of 3 -week-old $N$. benthamiana with pEAQ-HT-VP60 and pEAQ-HT-24K ${ }^{12}$ or by infiltration with the single plasmid, pEAQexpress-VP60-24K ${ }^{15}$. Leaves were harvested 5-7 days post infection. Wild type CPMV ${ }^{41}$ and $\mathrm{eVLPs}^{29}$ were extracted and purified (described below).

CPMV and eVLP particles were purified by the following method; leaf tissue was homogenized in a blender in 3 volumes of $0.1 \mathrm{M}$ sodium phosphate buffer, $\mathrm{pH}$ 7.0. The homogenate was filtered through two layers of muslin and clarified by centrifugation at $13,000 \mathrm{~g}$, for $20 \mathrm{~min}$ at $4{ }^{\circ} \mathrm{C}$. Virus particles were precipitated using 0.25 volumes of $20 \%$ (w/v) PEG6000 and $1 \mathrm{M} \mathrm{NaCl}$ and pelleted by centrifugation at $13,000 \mathrm{~g}$ for $20 \mathrm{~min}$ at $4{ }^{\circ} \mathrm{C}$. The resulting pellet was resuspended thoroughly in $10 \mathrm{mM}$ sodium phosphate, $\mathrm{pH}$ 7.0. eVLPs were purified further by centrifugation at $27,000 \mathrm{~g}$ for $20 \mathrm{~min}$ at $4{ }^{\circ} \mathrm{C}$ followed by a centrifugation of the clarified supernatant at $118,700 \mathrm{~g}$ for $2.5 \mathrm{~h}$ at $4{ }^{\circ} \mathrm{C}$. The pellet was resuspended in small volume of $10 \mathrm{mM}$ sodium phosphate buffer overnight at $4^{\circ} \mathrm{C}$. The eVLP suspension was further purified by centrifugation at $16,000 \mathrm{~g}$ for $15 \mathrm{~min}$ at $4^{\circ} \mathrm{C}$. For wild-type $\mathrm{CPMV}$ and mutated CPMV, the individual components were separated by centrifugation on $30-60 \%(\mathrm{w} / \mathrm{v})$ Nycodenz gradients ${ }^{42}$.

Mutagenesis. Point mutations were introduced into the coat protein-coding region of either pEAQ-HT-VP60 or pEAQ-RNA-2, a plasmid containing a full-length copy of CPMV RNA-2 in pEAQ-HT using the GENEART Site-Directed Mutagenesis System (Invitrogen) according to manufacturer's protocol. Primers for site-directed mutagenesis were designed using QuikChange Primer Design Program (Supplementary Table 4). To produce eVLPs containing the required mutation(s), the mutant forms of pEAQ-HT-VP60 were infiltrated into N. benthamiana in the presence of pEAQ-HT- $24 \mathrm{~K}^{12}$ and mutant eVLPs, if present, were extracted. To examine the effect of the mutations on virus infectivity and RNA encapsidation, the mutant forms of pEAQ-RNA-2 were co-infiltrated into $N$. benthamiana with $\mathrm{pBinP}-\mathrm{S} 2 \mathrm{NT}$ and any particles produced were purified as described for wild-type CPMV (above).

Deletions in the C-terminal region of the $\mathrm{S}$ subunit of eVLPs were made by PCR amplification using pEAQ-HT-VP60 as a template ${ }^{12}$. The primers used for PCR also contained appropriate restriction sites (BspEI and StuI) to replace the full-length VP60 with deleted variants of S subunit in pEAQ-HT-VP60 (Supplementary Table 5). The resulting deletion mutants were verified by sequencing before agroinfiltration of $N$. benthamiana plants in the presence of pEAQ-HT-24K. The formation of assembled capsids was assessed as described above. Raw images of uncropped gels are shown in Supplementary Figs 2 and 3).

Grid preparation and imaging. Cryo-EM grids were prepared by placing $3 \mu \mathrm{l}$ of $\sim 4.2 \mathrm{mg} \mathrm{ml}^{-1}$ (eVLP) or $\sim 5.8 \mathrm{mg} \mathrm{ml}^{-1}$ (CPMV-B) onto 200 mesh grids with $2-\mu \mathrm{m}$ holes (Quantifoil R2/2, Quantifoil Micro Tools, GmbH, Germany). Grids were glow discharged for $\sim 20 \mathrm{~s}$ prior plunge freezing in liquid ethane cooled by liquid nitrogen, using a FEI Vitrobot IV at $100 \%$ relative humidity. Data was collected on an FEI Titan Krios (MRC-LMB, Cambridge, UK.) transmission electron microscope at $300 \mathrm{kV}$, using an electron dose of $\sim 45 \mathrm{e}^{-}$per $\AA^{2}$ and a magnification of $\times 134,615$. The final object sampling was therefore of $1.04 \AA$ per pixel. A total of 1,135 (eVLP) and 1,754 (CPMV-B) exposures were recorded using the EPU automated acquisition software on a $17 \mathrm{~Hz}$ FEI Falcon II direct electron detector. Each exposure movie had a total exposure of $2 \mathrm{~s}$ and contained 35 images.

Image processing. Drift-corrected averages of each movie were created using MOTIONCORR ${ }^{30}$ and the contrast transfer function of each determined using CTFFIND3 (ref. 43); any images showing signs of astigmatism were discarded. All subsequent image processing steps were performed using RELION (v1.3) ${ }^{44}$ unless otherwise stated. Approximately 1,000 particles were manually picked and classified using reference-free $2 \mathrm{D}$ classification. The resulting $2 \mathrm{D}$ class average views were used as templates for automated particle picking ${ }^{31}$ (see Table 1 for particle numbers at each processing step). To produce a structurally homogeneous subset of CPMV particles, this initial stack was reduced using a statistical particlesorting algorithm ${ }^{31}$, which excludes the particles that are least similar to the initial search references. The remaining particles were classified using reference-free 2D classification to yield a data set for 3D structure refinement that only includes isolated molecular views of CPMV (see Table 1). These data sets, which comprise $\sim 80 \%$ of all initially selected particles, were used to calculate initial $3 \mathrm{D}$ reconstructions at $\sim 3.5 \AA$ (eVLP) and $4.1 \AA$ (CPMV-B). We then searched within each data set for a subset of particle images with greater homogeneity and/or higher resolution, using sequential 3D classification steps with icosahedral symmetry imposed. Each of these steps split the data into two, and the class with the sharpest features and highest resolution was taken forward. The initial starting model for the eVLP structure was a sphere with a radius of $\sim 155 \AA$. For the CPMV-B structure, the eVLP structure filtered to $\sim 60 \AA$ resolution was used. Following 7 rounds of $3 \mathrm{D}$ classification for eVLP and 13 rounds for CPMV-B, the final data sets (consisting of $\sim 8-10 \%$ of the original data) were considered to be homogeneous and used to determine final $3 \mathrm{D}$ reconstructions ${ }^{45}$. To correct for mechanical drift, beam-induced movement and radiation damage, statistical movie processing and particle polishing procedures were implemented ${ }^{45}$. As CPMV particles are readily visible even in individual movie frames, a running average of three frames was used in the calculations. Post-processing was employed to appropriately mask the model, estimate and correct for the $B$-factor of the maps ${ }^{46}$. The final resolution was determined using the 'gold standard' Fourier shell correlation $(F S C=0.143$ ) criterion $^{44}$ as $3.04 \AA$ for eVLP and $3.44 \AA$ for CPMV-B (FSC curves are shown in Supplementary Fig. 4). Local resolution was estimated using the ResMap wrapper in RELION ${ }^{47}$.

Model building and refinement. The backbone of each polypeptide chain in a single asymmetric unit of the $3 \AA$ eVLP cryo-EM map was built de novo using the 'baton building' tool in $\operatorname{Coot}^{32}$. The sequence was manually entered and the model refined using the 'real space refinement tool' in $\mathrm{Coot}^{32}$. Prior to refinement, secondary structure restraints were applied using ProSMART ${ }^{48}$. Structure factors obtained from the Fourier transform of the experimental density were used to restrain the coordinates in REFMAC5 (ref. 33). After each refinement round non-ideal rotamers, bond angles and Ramachandran outliers were improved. The final model was assessed for quality using MolProbity ${ }^{49}$. The refined eVLP atomic model was docked into the $3.4 \AA$ CPMV-B map. Residues in the eVLP atomic model for which no density was observed in the CPMV-B map were deleted (residues 190-202 in S subunit) and amino acids resolved in CPMV-B but not eVLP were added and modelled (residues 184-189 in S subunit). External restraints (produced from the eVLP model) and secondary structure restraints were applied using ProSMART, the structure was refined in REFMAC5 and assessed for quality using MolProbity (Table 2). Figures were generated using Chimera ${ }^{50}$ and PyMOL ${ }^{51}$

\section{References}

1. Natarajan, P. et al. Exploring icosahedral virus structures with VIPER. Nat. Rev. Microbiol. 3, 809-817 (2005)

2. Jiang, P., Liu, Y., Ma, H.-C., Paul, A. V. \& Wimmer, E. Picornavirus morphogenesis. Microbiol. Mol. Biol. Rev. 78, 418-437 (2014).

3. Lin, T. \& Johnson, J. E. Structures of picorna-like plant viruses: implications and applications. Adv. Virus Res. 62, 167-239 (2003).

4. Lin, T. et al. The refined crystal structure of cowpea mosaic virus at $2.8 \AA$ resolution. Virology 265, 20-34 (1999).

5. Ochoa, W. F., Chatterii, A., Lin, T. \& Johnson, J. E. Generation and structural analysis of reactive empty particles derived from an icosahedral virus. Chem. Biol. 13, 771-778 (2006)

6. Chen, Z. G. et al. Protein-RNA interactions in an icosahedral virus at $3.0 \AA$ resolution. Science 245, 154-159 (1989).

7. Lin, T. et al. Structural fingerprinting: subgrouping of comoviruses by structural studies of red clover mottle virus to $2.4 \AA$ resolution and comparisons with other comoviruses. J. Virol. 74, 493-504 (2000).

8. MacFarlane, S. A., Shanks, M., Davies, J. W., Zlotnick, A. \& Lomonossoff, G. P. Analysis of the nucleotide sequence of bean pod mottle virus middle component RNA. Virology 183, 405-409 (1991).

9. Taylor, K. M., Spall, V. E., Butler, P. J. \& Lomonossoff, G. P. The cleavable carboxyl terminus of the small coat protein of cowpea mosaic virus is involved in RNA encapsidation. Virology 255, 129-137 (1999).

10. Lomonossoff, G. P. \& Johnson, J. E. The synthesis and structure of comovirus capsids. Prog. Biophys. Mol. Biol. 55, 107-137 (1991).

11. Cañizares, M. C., Taylor, K. M. \& Lomonossoff, G. P. Surface-exposed C-terminal amino acids of the small coat protein of Cowpea mosaic virus are required for suppression of silencing. J. Gen. Virol. 85, 3431-3435 (2004).

12. Saunders, K., Sainsbury, F. \& Lomonossoff, G. P. Efficient generation of cowpea mosaic virus empty virus-like particles by the proteolytic processing of precursors in insect cells and plants. Virology 393, 329-337 (2009).

13. Sainsbury, F., Saunders, K., Aljabali, A. A. A., Evans, D. J. \& Lomonossoff, G. P. Peptide-controlled access to the interior surface of empty virus nanoparticles. Chembiochem. 12, 2435-2440 (2011).

14. Steinmetz, N. F., Lin, T., Lomonossoff, G. P. \& Johnson, J. E. Structure-based engineering of an icosahedral virus for nanomedicine and nanotechnology. Curr. Top. Microbiol. Immunol. 327, 23-58 (2009). 
15. Montague, N. P. et al. Recent advances of Cowpea mosaic virus-based particle technology. Hum. Vaccin. 7, 383-390 (2011).

16. Dalsgaard, K. et al. Plant-derived vaccine protects target animals against a viral disease. Nat. Biotechnol. 15, 248-252 (1997).

17. Liu, L. et al. Cowpea mosaic virus-based systems for the production of antigens and antibodies in plants. Vaccine 23, 1788-1792 (2005).

18. Steinmetz, N. F., Lomonossoff, G. P. \& Evans, D. J. Cowpea mosaic virus for material fabrication: addressable carboxylate groups on a programmable nanoscaffold. Langmuir 22, 3488-3490 (2006).

19. Steinmetz, N. F., Calder, G., Lomonossoff, G. P. \& Evans, D. J. Plant viral capsids as nanobuilding blocks: construction of arrays on solid supports. Langmuir 22, 10032-10037 (2006).

20. Steinmetz, N. F. et al. Virus-templated silica nanoparticles. Small 5, 813-816 (2009).

21. Shah, S. N., Steinmetz, N. F., Aljabali, A. A. A., Lomonossoff, G. P. \& Evans, D. J. Environmentally benign synthesis of virus-templated, monodisperse, ironplatinum nanoparticles. Dalton Trans. 28, 8479-8480 (2009).

22. Aljabali, A. A. A., Barclay, J. E., Butt, J. N., Lomonossoff, G. P. \& Evans, D. J. Redox -active ferrocene -modified Cowpea mosaic virus nanoparticles. Dalton Trans. 39, 7569-7574 (2010).

23. Aljabali, A. A. A., Barclay, J. E., Lomonossoff, G. P. \& Evans, D. J. Virus templated metallic nanoparticles. Nanoscale 2, 2596-2600 (2010).

24. Aljabali, A. A. A., Lomonossoff, G. P. \& Evans, D. J. CPMV-polyelectrolytetemplated gold nanoparticles. Biomacromolecules 12, 2723-2728 (2011).

25. Aljabali, A. A. A., Barclay, J. E., Steinmetz, N. F., Lomonossoff, G. P. \& Evans, D. J. Controlled immobilisation of active enzymes on the cowpea mosaic virus capsid. Nanoscale 4, 5640-5645 (2012).

26. Aljabali, A. A. A., Shukla, S., Lomonossoff, G. P., Steinmetz, N. F. \& Evans, D. J. CPMV-DOX delivers. Mol. Pharm. 10, 3-10 (2012).

27. Cañizares, M. C., Lomonossoff, G. P. \& Nicholson, L. Development of cowpea mosaic virus-based vectors for the production of vaccines in plants. Exp. Rev. Vaccines 4, 687-697 (2005).

28. Sainsbury, F., Thuenemann, E. C. \& Lomonossoff, G. P. pEAQ: versatile expression vectors for easy and quick transient expression of heterologous proteins in plants. Plant Biotechnol. J. 7, 682-693 (2009).

29. Sainsbury, F. et al. Genetic engineering and characterization of Cowpea mosaic virus empty virus-like particles. Methods Mol. Biol. 1108, 139-153 (2014).

30. Li, X. et al. Electron counting and beam-induced motion correction enable near-atomic-resolution single-particle cryo-EM. Nat. Methods 10, 584-590 (2013).

31. Scheres, S. H. W. Semi-automated selection of cryo-EM particles in RELION1.3. J. Struct. Biol. 189, 114-122 (2015).

32. Emsley, P., Lohkamp, B., Scott, W. G. \& Cowtan, K. Features and development of Coot. Acta Crystallogr. D Biol. Crystallogr. 66, 486-501 (2010).

33. Murshudov, G. N. et al. REFMAC5 for the refinement of macromolecular crystal structures. Acta Crystallogr. D Biol. Crystallogr. 67, 355-367 (2011).

34. Toropova, K., Basnak, G., Twarock, R., Stockley, P. G. \& Ranson, N. A. The three-dimensional structure of genomic RNA in bacteriophage MS2: implications for assembly. J. Mol. Biol. 375, 824-836 (2008).

35. Bakker, S. E. et al. Limits of structural plasticity in a picornavirus capsid revealed by a massively expanded equine rhinitis A virus particle. J. Virol. 88, 6093-6099 (2014).

36. Lander, G. C. et al. The structure of an infectious P22 virion shows the signal for headful DNA packaging. Science 312, 1791-1795 (2006).

37. Tang, L. et al. The structure of pariacoto virus reveals a dodecahedral cage of duplex RNA. Nat. Struct. Biol. 8, 77-83 (2001).

38. Johnson, K. N., Tang, L., Johnson, J. E. \& Ball, L. A. Heterologous RNA encapsidated in Pariacoto virus-like particles forms a dodecahedral cage similar to genomic RNA in wild-type virions. J. Virol. 78, 11371-11378 (2004).

39. Lin, T., Cavarelli, J. \& Johnson, J. E. Evidence for assembly-dependent folding of protein and RNA in an icosahedral virus. Virology 314, 26-33 (2003).

40. Liu, L. \& Lomonossoff, G. P. Agroinfection as a rapid method for propagating Cowpea mosaic virus-based constructs. J. Virol. Methods 105, 343-348 (2002).

41. Van Kammen, A. Purification and properties of the components of cowpea mosaic virus. Virology 31, 633-642 (1967).

42. Holness, C. L., Lomonossoff, G. P., Evans, D. \& Maule, A. J. Identification of the initiation codons for translation of cowpea mosaic virus middle component
RNA using site-directed mutagenesis of an infectious cDNA clone. Virology 172, 311-320 (1989).

43. Mindell, J. A. \& Grigorieff, N. Accurate determination of local defocus and specimen tilt in electron microscopy. J. Struct. Biol. 142, 334-347 (2003).

44. Scheres, S. H. W. \& Chen, S. Prevention of overfitting in cryo-EM structure determination. Nat. Methods 9, 853-854 (2012).

45. Scheres, S. H. W. RELION: Implementation of a Bayesian approach to cryo-EM structure determination. J. Struct. Biol. 180, 519-530 (2012).

46. Chen, S. et al. High-resolution noise substitution to measure overfitting and validate resolution in $3 \mathrm{D}$ structure determination by single particle electron cryomicroscopy. Ultramicroscopy 135, 24-35 (2013).

47. Kucukelbir, A., Sigworth, F. J. \& Tagare, H. D. Quantifying the local resolution of cryo-EM density maps. Nat. Methods 11, 63-65 (2014).

48. Nicholls, R. A., Long, F. \& Murshudov, G. N. Low-resolution refinement tools in REFMAC5. Acta Crystallogr. D Biol. Crystallogr. 68, 404-417 (2012).

49. Chen, V. B. et al. MolProbity: all-atom structure validation for macromolecular crystallography. Acta Crystallogr. D Biol. Crystallogr. 66, 12-21 (2009).

50. Pettersen, E. F. et al. UCSF Chimera-A visualization system for exploratory research and analysis. J. Comput. Chem. 25, 1605-1612 (2004).

51. Schrodinger, L. L. C. The PyMOL Molecular Graphics System, version 1.3r1 http://pymol.org (2010).

\section{Acknowledgements}

This work was supported by the UK Biotechnological and Biological Sciences Research Council (BBSRC) Grant BB/L020955/1, the Institute Strategic Programme Grant 'Understanding and Exploiting Plant and Microbial Secondary Metabolism' (BB/ J004596/1) and the John Innes Foundation. We thank the Wellcome Trust for equipment funding to support EM in Leeds (090932/Z/09/Z and 094232/Z/10/Z) and $\mathrm{PhD}$ studentship (R.F.T. 096685/Z/11/Z; K.C.D. 086774/Z/08/Z). We thank Shaoxia Chen and colleagues at the MRC-LMB (Cambridge, UK) for access to their Titan Krios microscope that made this study possible. N.A.R. had full access to all the data in the study and takes responsibility for the integrity of the data and the accuracy of the data analysis.

\section{Author contributions}

E.L.H., K.C.D., P.S., G.P.L. and N.A.R. conceived and designed the experiments; K.C.D., Y.M., P.S. and R.F.T. performed the experiments; E.L.H., Y.M., J.J.C., G.P.L. and N.A.R. analysed the data; E.L.H., Y.M., G.P.L. and N.A.R. wrote the paper.

\section{Additional information}

Accession codes: Coordinates are deposited in the Protein Data Bank under accession code 5A33 for CPMV eVLP and 5A32 for CPMV-B. Cryo-EM reconstructions are deposited in the EM Data Bank under accession codes EMD-3014 CPMV eVLP and EMD-3013 for CPMV-B.

Supplementary Information accompanies this paper at http://www.nature.com/ naturecommunications

Competing financial interests: G.P.L. declares that he is a named inventor on granted patent WO 29087391 Al which describes the transient expression system used in this manuscript to express eVLPs and also a named inventor on a filed patent 'Production of viral capsids' PBL. US 61/186,970 which describes the production and uses of CPMV eVLPs.

Reprints and permission information is available online at http://npg.nature.com/ reprintsandpermissions/

How to cite this article: Hesketh, E. L. et al. Mechanisms of assembly and genome packaging in an RNA virus revealed by high-resolution cryo-EM. Nat. Commun. 6:10113 doi: 10.1038/ncomms10113 (2015).

This work is licensed under a Creative Commons Attribution 4.0 International License. The images or other third party material in this article are included in the article's Creative Commons license, unless indicated otherwise in the credit line; if the material is not included under the Creative Commons license, users will need to obtain permission from the license holder to reproduce the material. To view a copy of this license, visit http://creativecommons.org/licenses/by/4.0/ 